\title{
Service Migration Algorithm Based On Markov Decision Process With Multiple QoS Attributes
}

Anhua Ma ( $\nabla$ manshmily@qq.com )

Nanjing University of Posts and Telecommunications https://orcid.org/0000-0002-1263-9587

Su Pan

Nanjing University of Posts and Telecommunications

Shuai Tao

Nanjing University of Posts and Telecommunications

Weiwei Zhou

Nanjing University of Posts and Telecommunications

\section{Research Article}

Keywords: Service migration, Markov decision process, QoS, SDN

Posted Date: October 1st, 2021

DOI: https://doi.org/10.21203/rs.3.rs-827252/v1

License: (9) This work is licensed under a Creative Commons Attribution 4.0 International License.

Read Full License 


\title{
Service migration algorithm based on Markov decision process with multiple QoS attributes
}

\author{
Anhua MA*1 ${ }^{* 1}$, Su PAN, Shuai TAO, Weiwei ZHOU \\ (Key Lab of Broadband Wireless Communication and Sensor Network Technology of Ministry of \\ Education, Nanjing University of Posts and Telecommunications, Nanjing 210003, China)

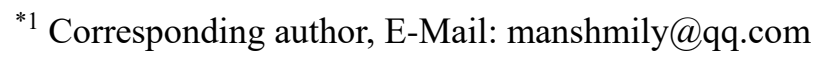

\begin{abstract}
With the rapid development of mobile internet cloud computing, the traditional network structure becomes non-suitable for advanced network traffic requirements. A service migration decision algorithm is proposed in the Software Defined Network(SDN) to satisfy differential Quality of Service(QoS) requirements. We divide services into real-time ones and non-real-time ones due to their different requirements on time delay and transmission rates, and construct the revenue function on two QoS attributes i.e. time delay and available transmission rates. We use the Markov decision process to maximize the overall benefits of users and network system to achieve the best user experience. The simulation results show that our proposed algorithm achieves better performance in terms of overall benefits than the exiting algorithms only considering single service and single QoS attribute.
\end{abstract}

Key words: Service migration, Markov decision process, QoS, SDN

\section{Introduction}

In recent years, mobile traffic grows geometrically with the development of cloud computing technology. At the same time, the QoS requirements of users increase rapidly, which challenge the communication network. The centralized communication network structure has a poor matching ability to advanced communication services. For example, when a user has moved a far distance away from the origin cloud server where the service starts, keeping communication with the origin server will cause the waste of network resources and a higher communication time delay, which leads to a bad user experience. In order to solve this series of problems, the Software Defined Network(SDN) architecture is used in the $5 \mathrm{G}$ network. The SDN technology decouples software from hardware of the network devices, which makes the functions of network devices no longer depend on dedicated hardware so that the resources can be fully and flexibly shared. The network matching ability can thereby be enhanced to satisfy various QoS requirements.

To solve the time delay problem caused by the user moving under the SDN architecture, the concept of 'Follow-me Cloud' was proposed in [5] [6] and [7]. Follow-me Cloud deploys data centers distributedly in various service areas, and the network migrates user services between servers in different areas according to the changes of user location, which keeps the users always communicating with the most suitable server. Compared with the stationary communication anchor point in the existing core network, the Follow-me Cloud can better 
guarantee the users' service experience and can reduce the end-to-end time delay between users and servers [6].

There are many researchers focus on the migration of services, in [7], the author introduced the basic implementation process of service migration in Follow-me Cloud. The authors in [8] described the issue of changes of user's IP address, the problem of migration trigger, and the process of signaling exchange in service migration in detail. To decide whether the user service should be migrated is the key research point. To solve this decision problem, the search-based dynamic migration algorithm was used in [9] to decide whether the service should be migrated to minimize service overhead. All of above researchers use greedy algorithm to solve the migration problem.

However, service migration decision is not just a single decision problem, since the user terminal is moving constantly. Each enough long distance move leads to a service migration decision, which belongs to the sequential decision-making problem. The greedy algorithm always makes the choice that seems to be the best at the current condition, which means it doesn't solve the problem in a global view. Thus, the decision it makes is the local optimal solution. i.e. each time that a user makes a move, the algorithm finds an optimal solution (migrate the service or not) according to the decision attributes at the current condition. However, this policy can not guarantee the overall benefits of whole user moving process.

Markov decision process calculates the benefit at the current decision moment and possible benefit at the next decision moment iteratively, which can guarantee the overall benefits after each step of decisions. Currently, only several works use the Markov decision process to solve service migration problems. The authors in [10] made service migration decisions by following the movement of a single user. However, it only considers user moving model on one dimension, which abstracts the user moving trail to a line. The user can only move along the line and it only uses a simple linear function combine the decision influence parameters including the distance between the user and start point and service migration overhead. The authors in [11] focus on finding the proper distance threshold according to user benefits and network overhead based on the work in [10]. The service migration would not occur when the user is within the threshold, and the migration happens when the user exceeds the threshold. Compared with work [10] and [11], the authors in [12] considered the two-dimensional user moving model to enrich the revenue function for the user.[12] reduce the complexity of the decision algorithm by making improvements on computation based on value iteration. The authors in [13] focused on following a stable server when multiple users request to migrate in or out, which makes service migration decisions according to the resource condition of the server and user experience. The authors in [22] analyzed the scenario of the vehicle edge network, makes the migration decision using revenue function combine network performance, the processing capacity of edge server, and movement status of the endpoint.

All the above works did not consider about two problems. The first is the impact of the difference of services. Multiple services usually run simultaneously in a user terminal, including real-time services with high-quality 
requirements for time delay and transmission rates, like video streams and mobile games, and non-real-time services like E-mail, FTP file transmission, etc. Different service types have different influences on migration decisions. Real-time services need to be migrated to get a better user experience along with user moving, while non-real-time services need not be migrated to prevent system overhead increasing. Thus, the service types should be considered. Secondly, the construction of revenue function in all the works above are quite simple, most of which are linear or non-linear functions only related to the distance between user and origin server, which are not practical enough since it does not consider the actual user QoS, such as time delay, throughput, available transmission rates, and network jitter, etc. For service migration decisions, these attributes of the QoS should be taken into consideration.

For the above reason, this paper proposed a QoS differentiated service migration decision algorithm based on the Markov decision process under SDN architecture. We divide services into real-time ones and non-real-time ones for their different requirements on time delay, we construct state space according to service types and the distance between user and origin server. We construct the revenue function based on two QoS attributes: time delay and available transmission rates. These two attributes have different weights on benefits according to real-time and non-real-time services. The purpose is to find an optimal policy to maximize the comprehensive overall benefits considering user experience and network overhead within a limited moving range of users.

\section{System model}

\subsection{Application scenario}

We consider wireless cellular network under SDN architecture shown in Fig.1 that each cell corresponds to a service area with a server. As shown in Fig.2, when the user terminal moves from service area 1 to service area 2 , it will send a service migration request to server 2 in service area 2 . The united cloud formed by these distributed servers will make a trade-off between the QoS requirement and the system overhead for service migration. A decision will be made whether the current server should migrate service to others If the migration request has been accepted, the service data will be migrated from server 1 to server 2. Otherwise, the user keeps communicating with server 1 .

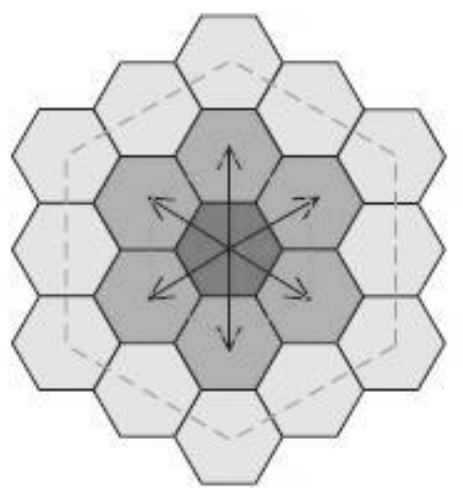

Fig.1 Wireless SDN cellular network 


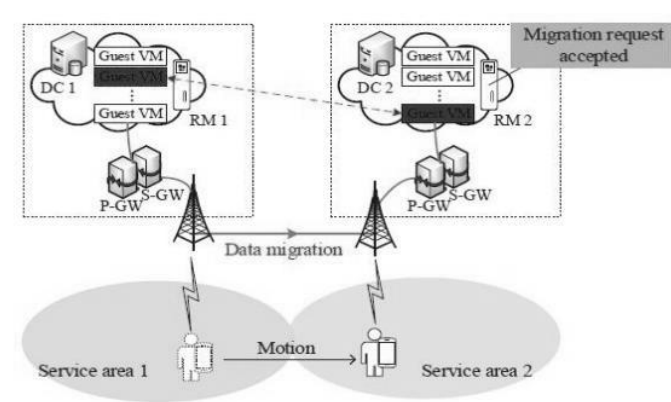

(a) Migration request accepted.

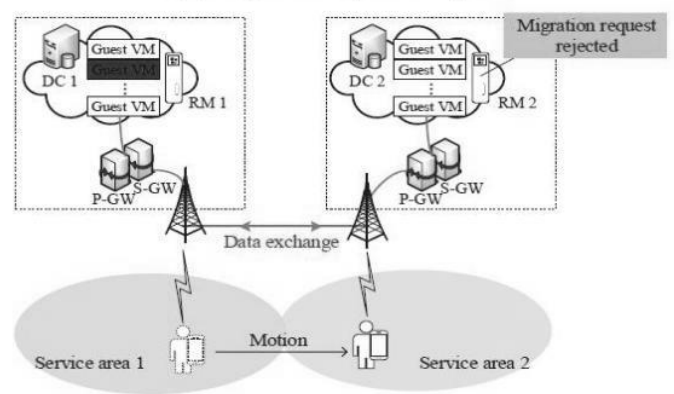

Fig.2 Process of service migration

\subsection{Markov decision model}

\subsubsection{Define the state space}

The changes of different Markov states lead to different instant benefits, so the state should reflect instant information. In the scenario considered in this paper, the user is moving continuously, the distance between the user's current cell and the origin cell is changing in real-time, and the services run on the user's terminal are also changing in real-time. So we define the two primary factors as the state space of the decision model as follows:

$$
s=\left\{D, s_{r}, s_{n}\right\} \in S,
$$

where $D \in\{0,1,2 . . t h r\}$ represents the distance from the user's current cell to the original cell in terms of circles, and thr represents the maximum circles from the user's current cell to the origin cell, when exceeding this distance service migration will be performed by default. $s_{r} \in\{0,1\}$ represents real-time services that have high requirements on time delay, such as video or audio stream and voice call, where 1 represents the user terminal is running this service, and 0 represents the user terminal is not running this service. $S_{n}$ represents non-real-time services such as background downloading, e-mail message receiving, where 0 and 1 have the same meaning as that for real-time services.

\subsubsection{Define the action}

The action is the decision made according to the revenue function under the current condition. It makes the state transferred from $s$ to $s^{\prime}$ through action $a$.

$$
a\left\{\begin{array}{l}
=1, a 1 \\
=0, a 0
\end{array}\right.
$$

When action $a$ equals 0 , the real-time and non-real-time services do not migrate. When action $a$ equals 1 , the real-time and non-real-time services migrate. In this paper, we assume that the real-time services and non-real-time services migrate simultaneously or not.

\subsubsection{Define the state transition probability}

To calculate the overall benefits by summing instant benefits of state space, we need to know the probability of each state. If the current state is already known, then we can calculate the probability of each state through state space transition probability since the next state is associated with the current one. As described above, the state space is divided by the distance and the service types of the user terminal. 
We evaluate the transition probability between the states of service types first, there are four states of real-time and non-real-time services on the user terminal, we transform the status of processing real-time services $s_{r}$ and non-real-time services $s_{n}$ into the state transition diagram shown in Fig.3.

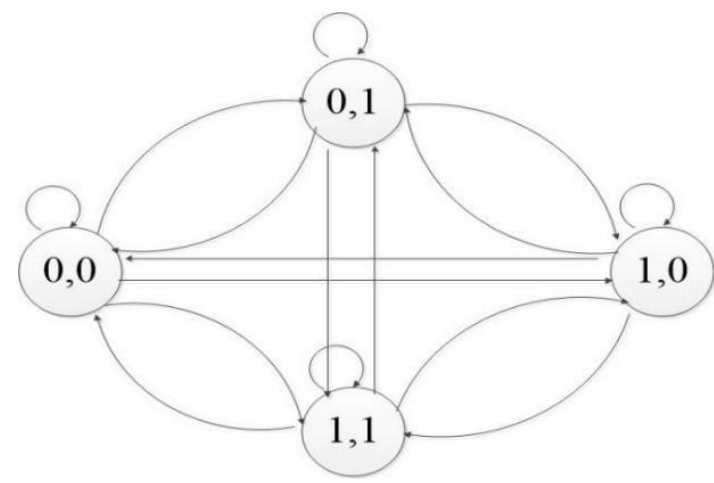

Fig.3 State transition diagram of real-time and non-real-time services
Assume that the arrival and departure of services follow the Poisson distribution, and the user terminal handles only one service request at a time. In other words, only one service enters or leaves the user terminal. The arrival and departure rates of real-time services are $\lambda_{1} e^{-\lambda_{1}}$ and $\mu_{1} e^{-\mu_{1}}$ respectively, the probability of keeping the same state is $1-\lambda_{1} e^{-\lambda_{1}}-\mu_{1} e^{-\mu_{1}}$. Similarly, the arrival and departure rates of non-real-time services are $\lambda_{2} e^{-\lambda_{2}}$ and $\mu_{2} e^{-\mu_{2}}$ respectively, the probability of keeping the same state is $1-\lambda_{2} e^{-\lambda_{2}}-\mu_{2} e^{-\mu_{2}}$. Then the transition probability matrix of real-time and non-real-time services of the user terminal is:

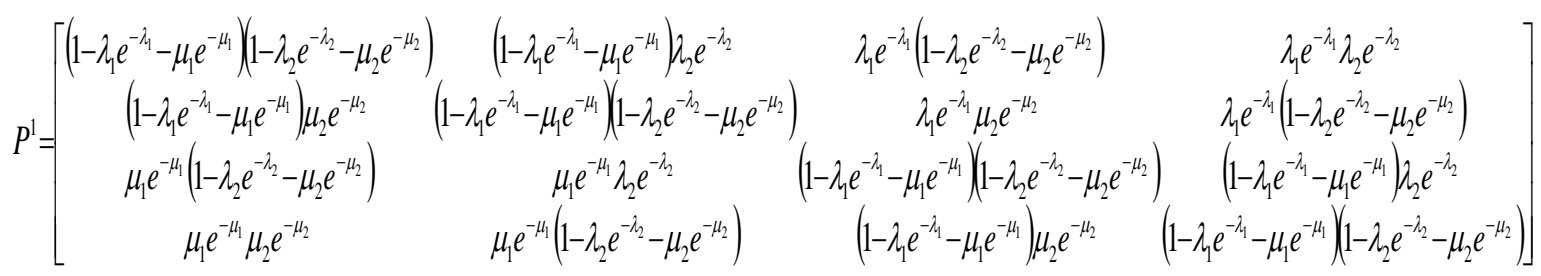

In transition probability matrix $P^{1}, P_{i, j}^{1}$ represents the probability of transition from the $i_{\text {th }}$ state to the $j_{\text {th }}$ state, where $i, j=1,2,3,4$ corresponds to the states of $\{0,0\},\{0,1\},\{1,0\},\{1,1\}$ in $\left\{s_{r}, s_{n}\right\}$. For example, $P_{1,1}^{1}$ represents the probability of the service state transfers from $\{0,0\}$ to $\{0,0\}$, which is the probability that real-time and non-real-time service status remain the same with the probability of

$$
\left(1-\lambda_{1} e^{-\lambda_{1}}-\mu_{1} e^{-\mu_{1}}\right)\left(1-\lambda_{2} e^{-\lambda_{2}}-\mu_{2} e^{-\mu_{2}}\right)
$$

Another example is that $P_{1,2}^{1}$ represents the probability of the service state transfers from $\{0,0\}$ to $\{0,1\}$, which is the probability of the user terminal starts a new non-real-time service while real-time service status remaining the same with the probability of $\left(1-\lambda_{1} e^{-\lambda_{1}}-\mu_{1} e^{-\mu_{1}}\right) \lambda_{2} e^{-\lambda_{2}}$.

The transition probability above is just 
real-time service $s_{r}$ and non-real-time service $s_{n}$ in state space. The state space also includes the state of circle distance $D$ between the user's current cell and the origin cell. Next, we evaluate the transition probability of circle distance $D$, Fig.4 shows the state transition diagram of circle distance D.

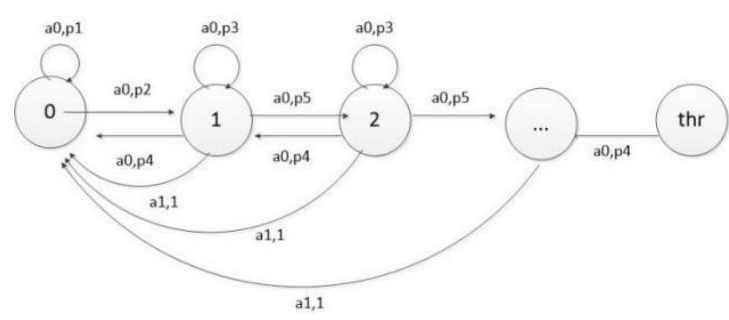

Fig.4 State transition diagram of circle distance

In the above diagram, 0 represents the user is in the origin cell, 1, 2 and other values represent the circle distance between the current cell and the origin cell. When taking action $a 0$, the service would not be migrated, and when taking action $a 1$, service migration will happen. After service migration, the $D$ in state space will return to 0 by default.

According to the cellular network architecture in the scenario we considered and the moving model research of the user terminal in a cellular network in [14], we can obtain the following user moving probability in the cellular network.

$$
\begin{aligned}
& p 1=1-p_{m} \\
& p 2=p_{m} \\
& p 3=1-p_{m}+\frac{2}{6} p_{m} \\
& p 4=\frac{1}{6} p_{m} \\
& p 5=\frac{3}{6} p_{m}
\end{aligned}
$$

where $p_{m}$ represents the probability of the user moving from the current cell to the neighboring cell.

From the state transition diagram and equation (4), we can get the transition probability matrix $P^{2}$ of the circle distance between the user's current cell and the origin cell, where $P_{i, j}^{2}$ represents the probability of transfer from state $i$ to state $j, i=0,1,2, \ldots, t h r$, $j=0,1,2, \ldots, t h r, i$ and $j$ represent the circle distance $D$ between the user's current cell and the origin cell. For example, $P_{0,0}^{2}$ represents the probability of circle distance changing from 0 to 0 , which is $p 1=1-p_{m}$ in the transition diagram. And the probability, such as $P_{0,2}^{2}$, will be set to 0 uniformly, which has not been defined in the transition diagram.

Combining the state transition probability matrix $P^{1}$ and $P^{2}$, we can get the global state transition probability matrix $P$ as:

$P=\left[\begin{array}{cccc}P^{1} * P_{0,0}^{2} & P^{1} * P_{0,1}^{2} & \ldots & P^{1} * P_{0, t h r}^{2} \\ P^{1} * P_{1,0}^{2} & P^{1} * P_{1,1}^{2} & \ldots & P^{1} * P_{1, t h r}^{2} \\ \ldots & \ldots & \ldots & \ldots \\ P^{1} * P_{t h r, 0}^{2} & P^{1} * P_{t h r, 1}^{2} & \ldots & P^{1} * P_{t h r, t h r}^{2}\end{array}\right]$

The size of the matrix is $4 *(t h r+1) \times 4 *(t h r+1)$, where $4 *(t h r+1)$ is the 
whole number of the state space, and the elements in the matrix are the probability $P\left[s^{\prime} \mid s, a\right]$ that a user transfers from state $s$ to state $s^{\prime}$ through action $a$.

\subsubsection{Instant benefit}

Instant revenue function depends on the current state, and a revenue function can be generated from a given state $s$. The specific instant revenue function is defined considering the following parts.

(1) The time delay benefits:

The user time delay is one of the important attributes that influence the QoS of user service. From the research on the time delay of user service in [15], we get the conclusion that the time delay of the user terminal is associated with the distance between itself and the server it makes data exchange with. We simplify the relationship between time delay and distance to be linear here when designing the revenue function. We define the distance $D$ to be 0 , and the user terminal has the minimum time delay of $t_{0}$ when the user is in the cell where the server locates. We get the time delay revenue function $t(s, a)$ through comprehensive consideration of both real-time services and non-real-time services as:

$$
t(s, a)=\left[s_{r}, s_{n}\right] \bullet\left[\alpha_{1} \frac{1}{t_{0}+k_{t} D}, \alpha_{2} \frac{1}{t_{0}+k_{t} D}\right]
$$

where $\alpha_{1}$ and $\alpha_{2}$ indicate the influence of time delay on real-time services and non-real-time services respectively. For real-time services with a high requirement for latency, $\alpha_{1}$ has a higher value, and for non-real-time services with a low requirement for latency, $\alpha_{2}$ has a lower value on the contrary. We set $t_{0}$ as the base time delay, which represents the time delay when the user receives data from the server in its' current cell. The user time delay is the sum of base time delay $t_{0}$ and additive time delay $k_{t} D$, where $D$ represents the circle distance between the user's current cell and the cell where the server that the user receives data locates. By different action $a$, different migrations lead to different $D$, hence the value of $D$ is associated with the current state $s$ and action a. $k_{t}$ represents the proportional coefficient of the time delay varying with the distance.

(2) Part of available rates benefit:

Now, we consider available rates, which is another attribute that influences the QoS of user service. Real-time services and non-real-times service of the user have their different transmission rate requirements. The user's benefit reaches the maximum when the service rates equal the rates the service requires. The data rate higher than the required rate will cause a waste of server resources, and the data rate lower than the required rate will lead to insufficient user experience. Combining the above demand relationship and refer to the relevant model in [16], we can get the available rates revenue function $b(s, a)$ :

$b(s, a)=\left[s_{r}, s_{n}\right]$

$$
\left[\beta_{1} \exp \left[-\frac{\left(x-\partial_{1}\right)^{2}}{2 \partial_{1}^{2}}\right], \beta_{2} \exp \left[-\frac{\left(x-\partial_{2}\right)^{2}}{2 \partial_{2}^{2}}\right]\right]
$$


where $\beta_{1}$ and $\beta_{2}$ represent the influences of available rates on real-time services and non-real-time services respectively, $x$ represents the data rates provided by the server, $\partial_{1}$ and $\partial_{2}$ represent the data rates required by real-time services and non-real-time services respectively, and the function $\exp \left[-\frac{\left(x-\partial_{1}\right)^{2}}{2 \partial_{1}^{2}}\right]$ is used to curve the benefit according to above description.

\section{(3) Cost:}

Last, we consider the consumption of resource by service migration in the core network. We simply assume that the consumption of migration of real-time and non-real-time service are fixed values respectively. We define the cost function $c(s, a)$ as:

$$
\begin{aligned}
c(s, a) & =\left[s_{r}, s_{n}\right] \bullet a * m \\
m & =\left[k_{m} C_{1}, k_{m} C_{2}\right] .
\end{aligned}
$$

The cost function is the product of state vector $\left[s_{r}, s_{n}\right]$ with action vector $a$ and cost vector $m$, among which the cost vector is $m=\left[k_{m} C_{1}, k_{m} C_{2}\right], C_{1}$ and $C_{2}$ represent the utilization of system resource while doing real-time and non-real-time services migration respectively, $k_{m}$ represents the weight coefficient of cost consumption to the overall benefits.

(4) Instant revenue function:

According to (6) and (7), we can get the revenue function:

$$
\begin{aligned}
& g(s, a)=t(s, a)+b(s, a) \\
& =\left[s_{r}, s_{n}\right] \bullet\left[\alpha_{1} \frac{1}{t_{0}+k_{t} D}, \alpha_{2} \frac{1}{t_{0}+k_{t} D}\right] \\
& +\left[s_{r}, s_{n}\right]\left[\beta_{1} \exp \left[-\frac{\left(x-\partial_{1}\right)^{2}}{2 \partial_{1}^{2}}\right], \beta_{2} \exp \left[-\frac{\left(x-\partial_{2}\right)^{2}}{2 \partial_{2}^{2}}\right]\right]
\end{aligned}
$$

Then, according to (8) and (9), we can get the final instant revenue function:

$$
r(s, a)=g(s, a)-c(s, a)
$$

$$
\begin{aligned}
& =\left[s_{r}, s_{n}\right]\left[\alpha_{1} \frac{1}{t_{0}+k_{t} D}, \alpha_{2} \frac{1}{t_{0}+k_{t} D}\right] \\
& +\left[s_{r}, s_{n}\right]\left[\beta_{1} \exp \left[-\frac{\left(x-\partial_{1}\right)^{2}}{2 \partial_{1}^{2}}\right], \beta_{2} \exp \left[-\frac{\left(x-\partial_{2}\right)^{2}}{2 \partial_{2}^{2}}\right]\right] \\
& -\left[s_{r}, s_{n}\right] \rho *\left[k_{m} C_{1}, k_{m} C_{2}\right]
\end{aligned}
$$

\subsubsection{Optimization model}

We have defined the instant revenue function above, the goal of our model is to maximize the overall benefits. The overall revenue function is:

$$
v^{\pi}\left(s^{0}\right)=E_{\pi}\left\{\sum_{t=0}^{\infty} \gamma^{t} r\left(s^{t}, a^{t}\right)\right\},
$$

where $\pi$ is a service migration decision policy constituted of a series of actions under specific states, the initial state is $s^{0}, \gamma \in[0,1]$ represents the reduction factor which means the importance of future benefits relative to instant benefits. Especially, $\gamma=0$ represents we only consider instant benefits without considering long-term benefits, while $\gamma=1$ 
represents we consider long-term benefits as important as instant benefits. $E_{\pi}\left\{\sum_{t=0}^{\infty} \gamma^{t} r\left(s^{t}, a^{t}\right)\right\}$ represents the overall revenue function through calculating the expectation of the instant revenue function.

Our goal is to find out a maximum overall revenue function $v^{\pi^{*}}\left(s^{0}\right)$ as follows:

$$
v^{\pi^{*}}\left(s^{0}\right)=\max _{a^{0} \in A} E_{\pi}\left\{\sum_{t=0}^{\infty} \gamma^{t} r\left(s^{t}, a^{t}\right)\right\}
$$

Lemma 1: The over all revenue $v^{\pi}\left(s^{0}\right)$ can be expressed as:

$$
\begin{aligned}
v^{\pi}\left(s^{0}\right) & =\sum_{s^{\prime} \in S} P\left[s^{\prime} \mid s^{0}, a^{0}\right] r\left(s^{0}, a^{0}\right) \\
& +\sum_{s^{\prime} \in S} \gamma P\left[s^{\prime} \mid s^{0}, a^{0}\right] v^{\pi}\left(s^{\prime}\right)
\end{aligned}
$$

Proof: For each state is associated with the previous state, so the overall revenue is related with the initial state and the actions of each state, we extract the first term from the expectation of (11), and obtain (14) as follows:

$$
v^{\pi}\left(s^{0}\right)=E_{\pi}\left\{r\left(s^{0}, a^{0}\right)+\gamma \sum_{t=0}^{\infty} \gamma^{t} r\left(s^{t+1}, a^{t+1}\right)\right\}
$$

We expand the first half of (14), it follows:

$$
E_{\pi}\left\{r\left(s^{0}, a^{0}\right)\right\}=\sum_{s^{\prime} \in S} P\left[s^{\prime} \mid s^{0}, a^{0}\right] r\left(s^{0}, a^{0}\right),
$$

and then transform the last half of (14) into (16):

$$
\begin{aligned}
& E_{\pi}\left\{\gamma \sum_{t=0}^{\infty} \gamma^{t} r\left(s^{t+1}, a^{t+1}\right)\right\} \\
= & \sum_{s^{\prime} \in S} P\left[s^{\prime} \mid s^{0}, a^{0}\right] \gamma E_{\pi}\left\{\sum_{t=0}^{\infty} \gamma^{t} r\left(s^{t+1}, a^{t+1}\right)\right\}
\end{aligned}
$$

$$
\text { Replace } E_{\pi}\left\{\sum_{t=0}^{\infty} \gamma^{t} r\left(s^{t+1}, a^{t+1}\right)\right\} \text { in (16) }
$$

with $v^{\pi}\left(s^{\prime}\right)$, and add (15) and (16), it follows:

$$
\begin{aligned}
v^{\pi}\left(s^{0}\right) & =\sum_{s^{\prime} \in S} P\left[s^{\prime} \mid s^{0}, a^{0}\right] r\left(s^{0}, a^{0}\right) \\
& +\sum_{s^{\prime} \in S} \gamma P\left[s^{\prime} \mid s^{0}, a^{0}\right] v^{\pi}\left(s^{\prime}\right)
\end{aligned}
$$

With the Lemma 1, (12) can be expressed as:

$$
\begin{aligned}
v^{\pi^{*}}\left(s^{0}\right)=\max _{a^{0} \in A}\left\{\sum_{s^{\prime} \in S} P\left[s^{\prime} \mid s^{0}, a^{0}\right] r\left(s^{0}, a^{0}\right)\right. \\
\left.+\sum_{s^{\prime} \in S} \gamma P\left[s^{\prime} \mid s^{0}, a^{0}\right] v^{\pi}\left(s^{\prime}\right)\right\}
\end{aligned}
$$

End of proof.

Different $\pi$ will lead to different values of overall revenue function by adopting different policies. Our goal is to find out a policy $\pi^{*}$ to maximize the summation of overall benefits for all states, and that is the migration algorithm we get last to make the user experience and network overhead optimized.

\subsection{Model solving}

This paper uses a numerical iterative method to solve the Markov decision model, the detailed process is as follows: 


\section{Numerical iterative algorithm:}

1. Initialize $v^{0}(s)=0, \varepsilon>0, k=0$;

\section{Repeat}

3. update $v^{k+1}(s)=\max _{a \in A}\left\{\sum_{s^{\prime} \in S} P\left[s^{\prime} \mid s^{0}, a^{0}\right] r(s, a)+\sum_{s^{\prime \prime} \in S} \gamma P\left[s^{\prime \prime} \mid s, a\right] v^{k}\left(s^{\prime \prime}\right)\right\}$;

4. update $k=k+1$;

5. Until $\left\|v^{k+1}-v^{k}\right\|<\varepsilon(1-\gamma) /(2 \gamma)$;

6. Return $\delta(s)=\arg \max _{a \in A}\left\{\sum_{s^{\prime} \in S} P\left[s^{\prime} \mid s^{0}, a^{0}\right] r(s, a)+\sum_{s^{\prime \prime} \in S} \gamma P\left[s^{\prime \prime} \mid s, a\right] v^{k}\left(s^{\prime \prime}\right)\right\}$

The numerical iterative method initializes the overall revenue function of each state to 0 at first and keeps updating the overall revenue function for each state until the overall revenue function converges to $v^{\pi^{*}}\left(s^{0}\right)$. The set $\pi$ of all actions $a$ which satisfies these optimized benefits is the optimized policy $\pi^{*}$ we need .

\section{Simulation and Discussions}

Assume the arrival rate and departure rate are $6.9^{*} 10^{-3}($ services $/ \mathrm{s})$ and $1.85 * 10^{-4}($ services $/ \mathrm{s})$ for real-time services, and $\quad 6 * 10^{-2}($ services $/ \mathrm{s}) \quad$ and $1.39 * 10^{-4}($ services $/ \mathrm{s})$ for non-real-time services. The probability $p_{m}$ that the terminal moves from the current cell to a neighbor cell around is 0.75 . The base time delay $t_{0}$ in the time delay revenue function is set as $30 \mathrm{~ms}$.
The required data rates of real-time and non-real-time services $\partial_{1}$ and $\partial_{2}$ are $20 \mathrm{Mbps}$ and 200Kbps respectively, and the data rates provided by the server for services (achievable data rates)are randoms in a certain range.

Based on the parameters set above, we perform simulations to compare differentiated QoS service migration decision algorithm proposed in this paper with single service migration decision algorithm(as comparison algorithm 1) and single QoS attribute service migration decision algorithm(as comparison algorithm 2).

For a user terminal, the cell location $D$ and service state $\left[s_{r}, s_{n}\right]$ of real-time and non-real-time services keep changing, so we consider that real-time and non-real-time 
services coexist in the terminal at the same time.

First, we compare the differentiated QoS service migration decision algorithm with a single service migration decision algorithm. Single service migration decision algorithm did not consider different influences on real-time and non-real-time services of various QoS attributes, which means the difference of influences on different services between different QoS attributes has not been distinguished. The compared simulation results are shown in Fig.5.

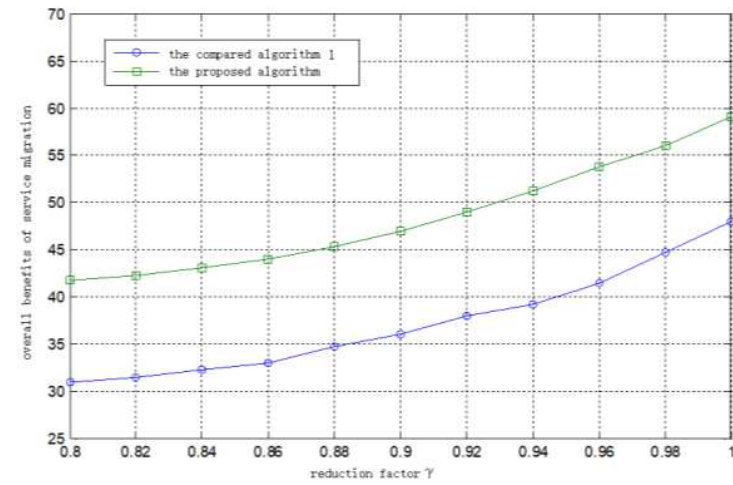

Fig.5 Changes of overall benefits of service migration with reduction factors under two algorithms

Fig.5 shows the changes of overall benefits under different values of reduction factor $\gamma$ within state space $\{D, 1,1\}$. With the increase of reduction factor, the overall benefits of service migration perform the increasing trend, which means long term benefits from all possible states after the current state gradually improve the effect on the overall benefits in the Markov decision process, the overall benefits reaches the maximum when $\gamma$ is equals to 1 . The result also proves that the algorithm proposed in this paper can improve user experience during service migration and reduce the overall cost of the network.

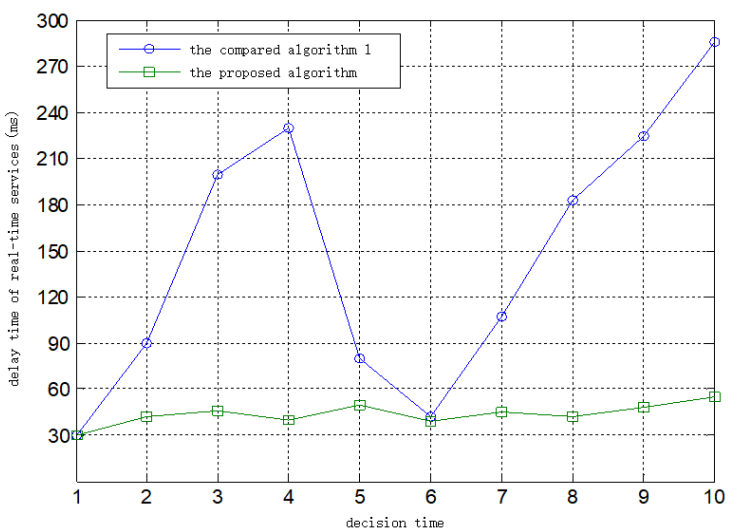

Fig.6 Changes of delay time under two algorithms

Fig.6 shows the changes of the delay time of real-time services under two algorithms when the user terminal moves. The decision time is when the system needs to decide whether to do service migration while the user terminal moves and $D$ changes. Fig.6 shows that since the differentiated QoS service migration decision algorithm proposed in this paper pays attention to the attribute of the delay time of real-time services, it can make migration in time to keep the delay time of real-time services at a low level. While single service migration decision algorithm does not distinguish the different requirements on the delay time of real-time and non-real-time services, which leads to large jitter on the delay time of real-time services. Compared with the two mentioned algorithms, the algorithm proposed in this paper can provide a better guarantee to the user experience of real-time service.

Next, we consider the situations of only real-time services existing or only non-real-time services existing with the state space of $\{D, 1,0\}$ and $\{D, 0,1\}$ respectively. We make a comparison between the 
differentiated QoS service migration decision algorithm proposed in this paper and a single QoS attribute service migration decision algorithm in [10]. The instant revenue function of differentiated QoS service migration decision algorithm here are $\alpha_{1} \frac{1}{t_{0}+k_{t} D}+\beta_{1} \exp \left[-\frac{\left(x-\partial_{1}\right)^{2}}{2 \partial_{1}^{2}}\right]-a^{*} k_{m} C_{1}$ and $\alpha_{2} \frac{1}{t_{0}+k_{t} D}+\beta_{2} \exp \left[-\frac{\left(x-\partial_{2}\right)^{2}}{2 \partial_{2}^{2}}\right]-a^{*} k_{m} C_{2}$ that correspond to situations of only real-time services existing and only non-real-time services existing, respectively. The single QoS attribute service migration decision algorithm only considers one single QoS attribute, i.e. the QoS attribute of time delay. Refer to [10], the instant revenue function can be simplified as $\quad q_{1}-k_{q 1} D-a^{*} k_{m} C_{1} \quad$ and $q_{2}-k_{q 2} D-a^{*} k_{m} C_{2}, \quad$ where $q_{1}$ and $q_{2}$ correspond to the maximum value of $\alpha_{1} \frac{1}{t_{0}+k_{t} D}$ and $\alpha_{2} \frac{1}{t_{0}+k_{t} D}$, which are the maximum time delay benefits of real-time service and non-real-time service when the user is in the cell where the server locates. $k_{q 1}$ and $k_{q 2}$ are the coefficients of the single QoS attribute that represent the decrease of QoS with distance increasing. By comparing the two algorithms, we get Fig.7 and Fig.8 as below.

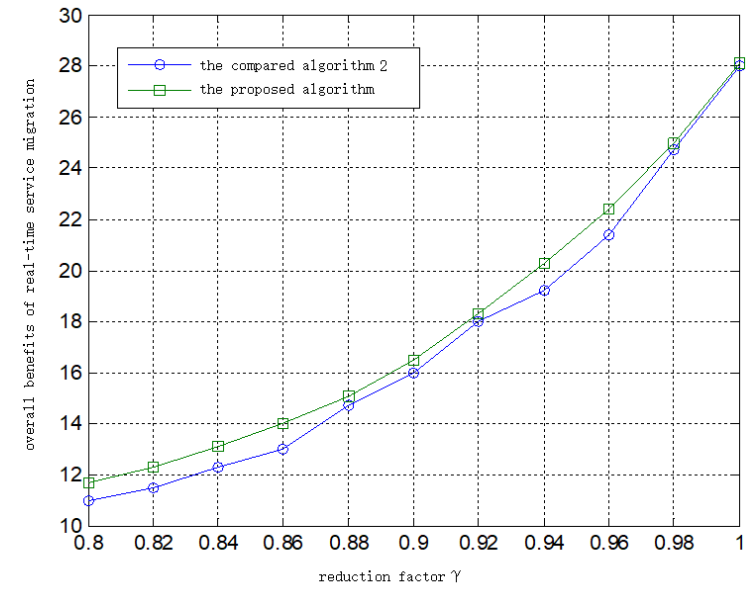

Fig.7 Changes of overall benefits of real-time service migration under two algorithms with the change of reduction factor

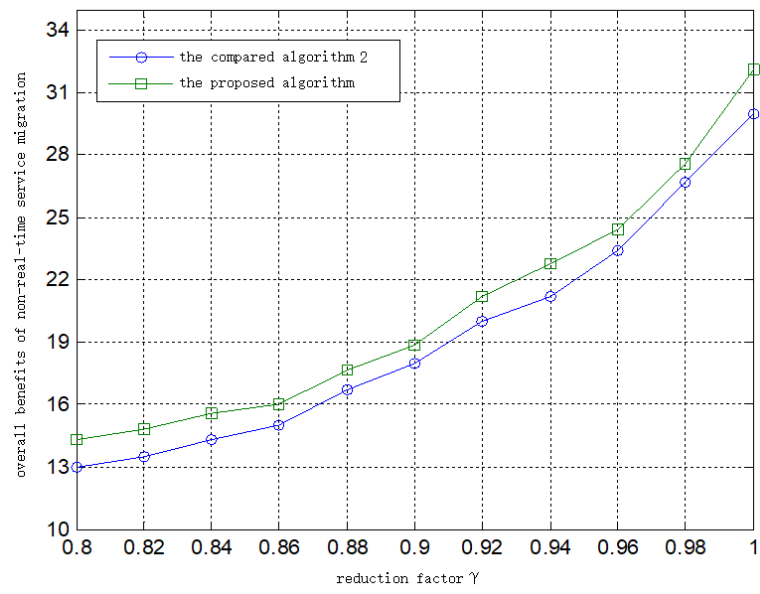

Fig.8 Changes of overall benefits of non-real-time service migration under two algorithms with the change of reduction factor

Fig.7 and Fig.8 show that the overall benefit obtained by the differentiated QoS service migration decision algorithm proposed in this paper is slightly better than the situation of the single QoS attribute one. That is because our algorithm provides an enriched revenue function constructed of time delay, available rates and migration cost, which means it has more parameters to be adjusted while doing MDP iterations. So the algorithm can make better balance between user 
experience and system overhead while making service migration decision, which leads to more overall benefits. Moreover, while making migration decision for non-real-time services, due to non-real-time services are not sensitive to time delay, the gain of our algorithm is more than what with the real-time services only situation.

\section{Conclusion}

This paper researches the problem of service types and QoS attributes that influence service migration decisions under SDN architecture. We propose a differentiated QoS service migration decision algorithm based on Markov decision process, we construct revenue function through several different QoS attributes and distinguishes different requirements of real-time services and non-real-time services from the user terminal. The simulation results show that the algorithm proposed in this paper can improve user experience under the situation of multi-service and multi-QoS attributes, and can reduce system overhead efficiently when conducting service migration.

\section{References}

[1] Wang C X, Haider F, Gao X, et al. Cellular architecture and key technologies for $5 \mathrm{G}$ wireless communication networks[J]. IEEE Communications Magazine, 2014, 52(2):122-130.

[2] Aissioui A, Ksentini A, Gueroui A M, et al. Toward Elastic Distributed SDN/NFV Controller for $5 \mathrm{G}$ Mobile Cloud Management Systems[J]. Access IEEE, 2016, 3:2055-2064.
[3] Matias J, Garay J, Toledo N, et al. Toward an SDN-enabled NFV architecture[J]. IEEE Communications Magazine, 2015, 53(4):187-193.

[4] Chartsias P K, Amiras A, Plevrakis I, et al. SDN/NFV-based end to end network slicing for $5 \mathrm{G}$ multi-tenant networks $[\mathrm{C}] / /$ European Conference on Networks and Communications. IEEE, 2017:1-5.

[5] Taleb T, Ksentini A, Frangoudis P. Follow-Me Cloud: When Cloud Services Follow Mobile Users[J]. IEEE Transactions on Cloud Computing, 2016, 99:1-1.

[6] Aissioui A, Ksentini A, Gueroui A. PMIPv6-Based Follow Me Cloud[C]// IEEE Global Communications Conference. IEEE, 2016:1-6.

[7] Taleb T. Toward carrier cloud: Potential, challenges, and solutions[J]. IEEE Wireless Communications, 2014, 21(3):80-91.

[8] Taleb T, Ksentini A. Follow me cloud: interworking federated clouds and distributed mobile networks[J]. Network IEEE, 2013, 27(5):12-19.

[9] Wang Y, Shi W. On service migration in the cloud to facilitate mobile accesses $[\mathrm{C}] / / \quad$ IEEE International Conference on CLUSTER Computing. IEEE, 2013:1-5.

[10] Ksentini A, Taleb T, Chen M. A Markov Decision Process-based service migration procedure for follow me cloud[C]// IEEE International Conference on Communications. IEEE, 
[11] Wang S, Urgaonkar R, He $\mathrm{T}$, et al. Mobility-Induced Service Migration in Mobile Micro-clouds[C]// Military Communications Conference. IEEE, 2014:835-840.

[12] Wang S, Urgaonkar R, Zafer M, et al. Dynamic service migration in mobile edge-clouds[C]// Ifip NETWORKING Conference. IEEE, 2015:1-9.

[13] Chen J, Long H, Zheng Q, et al. An SMDP-Based Resource Management Scheme for Distributed Cloud Systems[C]// Vehicular Technology Conference. IEEE, 2015:1-5.

[14] Chiang K H, Shenoy N. A 2-D Random-Walk Mobility Model for Location-Management Studies in Wireless Networks[J]. IEEE Transactions on Vehicular Technology, 2008, 53(2):413-424.

[15] Stiliadis D, Varma A. Latency-rate servers: a general model for analysis of traffic scheduling algorithms[C]// Fifteenth Joint Conference of the IEEE Computer and Communications Societies Conference on the Conference on Computer Communications. IEEE Computer Society, 1996:111-119.

[16] Su P, Zhou W, Gu Q, et al. Network Selection Algorithm Based on Spectral Bandwidth Mapping and an Economic Model in WLAN\&LTE heterogeneous networks[J]. KSII Transactions on Internet \& Information Systems, 2015, 9(1):68-86.

[17] Bifulco R, Canonico R. Analysis of the handover procedure in Follow-Me Cloud $[\mathrm{C}] / / \quad$ IEEE, International
Conference on Cloud NETWORKING. IEEE, 2013:185-187.

[18] Gomes A S, Fonseca V, Sousa B, et al. A mobile follow-me cloud content caching model[C]// Network Operations and Management Symposium. IEEE, 2016:763-766.

[19] Nadembega A, Hafid A S, Brisebois R. Mobility prediction model-based service migration procedure for follow me cloud to support QoS and QoE[C]// IEEE International Conference on Communications. IEEE, 2016:1-6.

[20] Ksentini A, Taleb T, Messaoudi F. A LISP-Based Implementation of Follow Me Cloud[J]. Access IEEE, 2014, 2:1340-1347.

[21] Wang S, Zafer M, Leung K K. Online Placement of Multi-Component Applications in Edge Computing Environments[J]. IEEE Access, 2017, 5:2514-2533.

[22] Guo H, Rui L L, Gao Z P. Dynamic service migration strategy based on MDP model with multiple parameter in vehicular edge network[J]. Journal on Communications, Vol. 41, No. 1, Jan 2020.

\section{Declarations}

Funding:

The National Natural Science Foundation of China (No. 61271235).

Conflicts of interest/Competing interests:

Not applicable. 
Availability of data and material:

Not applicable.

Code availability:

Not applicable.

Ethics approval:

Not applicable.

Consent of participate:

Not applicable.

Consent of publication:

Agree to authorize Telecommunication

Systems to publish this article. 